\title{
Robotic external ventricular drain placement for acute neurosurgical care in low-resource settings: feasibility considerations and a prototype design
}

\author{
Alexander D. Smith, BS, ${ }^{1}$ Alexander J. Teague, BS, ${ }^{1}$ Anant Naik, BS, ${ }^{1}$ Mika Janbahan, BS, ${ }^{1}$ \\ Emily J. Smith, BS, ${ }^{1}$ David T. Krist, PhD, ${ }^{1}$ Sindhu Parupalli, BS, ${ }^{1}$ Kevin Teal, MD, ${ }^{1,2}$ and \\ Wael Hassaneen, MD, PhD ${ }^{1,2}$
}

${ }^{1}$ Carle Illinois College of Medicine, University of Illinois at Urbana-Champaign, Urbana; and ${ }^{2}$ Department of Neurological Surgery, Neuroscience Institute, Carle Foundation Hospital, Urbana, Illinois

\begin{abstract}
OBJECTIVE Emergency neurosurgical care in lower-middle-income countries faces pronounced shortages in neurosurgical personnel and infrastructure. In instances of traumatic brain injury (TBI), hydrocephalus, and subarachnoid hemorrhage, the timely placement of external ventricular drains (EVDs) strongly dictates prognosis and can provide necessary stabilization before transfer to a higher-level center of care that has access to neurosurgery. Accordingly, the authors have developed an inexpensive and portable robotic navigation tool to allow surgeons who do not have explicit neurosurgical training to place EVDs. In this article, the authors aimed to highlight income disparities in neurosurgical care, evaluate access to CT imaging around the world, and introduce a novel, inexpensive robotic navigation tool for EVD placement.

METHODS By combining the worldwide distribution of neurosurgeons, CT scanners, and gross domestic product with the incidence of $\mathrm{TBI}$, meningitis, and hydrocephalus, the authors identified regions and countries where development of an inexpensive, passive robotic navigation system would be most beneficial and feasible. A prototype of the robotic navigation system was constructed using encoders, 3D-printed components, machined parts, and a printed circuit board.

RESULTS Global analysis showed Montenegro, Antigua and Barbuda, and Seychelles to be primary candidates for implementation and feasibility testing of the novel robotic navigation system. To validate the feasibility of the system for further development, its performance was analyzed through an accuracy study resulting in accuracy and repeatability within $1.53 \pm 2.50 \mathrm{~mm}$ (mean $\pm 2 \times \mathrm{SD}, 95 \% \mathrm{Cl})$.
\end{abstract}

CONCLUSIONS By considering regions of the world that have a shortage of neurosurgeons and a high incidence of EVD placement, the authors were able to provide an analysis of where to prioritize the development of a robotic navigation system. Subsequently, a proof-of-principle prototype has been provided, with sufficient accuracy to target the ventricles for EVD placement.

https://thejns.org/doi/abs/10.3171/2021.10.FOCUS21544

KEYWORDS neurosurgical navigation; global health; inexpensive robot; external ventricular drain; traumatic brain injury; hydrocephalus

$\mathrm{E}$ XTERNAL ventricular drains (EVDs) can provide lifesaving stabilization in patients with acute brain injury, including traumatic brain injury (TBI), acute hydrocephalus, and intracranial hemorrhage. ${ }^{1}$ EVDs allow for the measurement and management of increased intracranial pressure (ICP) caused by obstruction of CSF outflow. Additionally, EVD placement is indicated for temporary diversion of CSF, such as in acute hydrocephalus, when a permanent shunt cannot yet be placed; in the case of an infected preexisting shunt; or in meningitis, when repeated lumbar punctures do not provide adequate relief. EVDs can also be used for intrathecal medication administration. $^{2}$

In many conditions, placement of an EVD is a timecritical and necessary procedure. In TBI, EVDs drain CSF and any ventricular hemorrhage to lower ICP promptly and, later, serve as monitors of ICP, guiding future medical therapy. ${ }^{3}$ TBI affects up to 69 million individuals world-

ABBREVIATIONS EVD = external ventricular drain; GDP = gross domestic product; HIC = high-income country; ICP = intracranial pressure; LMIC = lower-middle-income country; ROS = Robot Operating System; TBI = traumatic brain injury.

SUBMITTED September 1, 2021. ACCEPTED October 18, 2021.

INCLUDE WHEN CITING DOI: 10.3171/2021.10.FOCUS21544. 
wide every year with mild, moderate, and severe degrees of severity. ${ }^{4}$ An estimated 1.6 million cases of TBI that require neurosurgery occur in high-income countries (HICs) per year, whereas an estimated 4.5 million cases occur in lower-middle-income countries (LMICs) per year. ${ }^{5}$

EVDs also provide benefits in the treatment of acute hydrocephalus. There are approximately 30,000 cases of hydrocephalus requiring neurosurgery in HICs and 740,000 cases in LMICs per year. ${ }^{5}$ In LMICs, EVDs are commonly placed for acute hydrocephalus secondary to CNS infections, which has repeatedly shown benefits. ${ }^{6-9}$ In infants, the most common cause of acquired hydrocephalus is intraventricular hemorrhage, and studies have shown that early intervention (at the age of 25 days or younger) with an EVD leads to better neurological outcomes. ${ }^{10,11}$ Improvements in cognitive, communicative, and social functioning have demonstrated that the timeliness of EVD placement can have a profound effect on outcomes. ${ }^{10,11}$

Accompanying the global need for timely EVD placement is a shortage of neurosurgeons. In the United States alone, there is approximately 1 neurosurgeon per 61,000 people; however, in 44 states, there are fewer than 2 neurosurgeons per 100,000 people. ${ }^{12}$ Additionally, in the United States there is not only a shortage of neurosurgeons, but also a predicted overall shortage of 130,600 physicians by 2025 . In LMICs, the shortage of physicians and surgical care is exacerbated by increased delays in seeking, reaching, and receiving care. In these regions, there are deficiencies in access to neurosurgical care. For example, in sub-Saharan Africa, there is 1 neurosurgeon for every 3.1 to 6.4 million people. ${ }^{13}$ Studies have shown that only $11.8 \%$ of necessary neurosurgical care is met in LMICs. ${ }^{14}$

There are limitations in access not only to neurosurgeons but also to CT scanners in many parts of the world. In sub-Saharan Africa, only $20.3 \%$ of the population lives within 2 hours of a facility that has both a CT scanner and a neurosurgeon. ${ }^{13}$ In East Asia and the Pacific, this percentage decreases to $17.4 \% .{ }^{13}$ Studies have shown that in LMICs, there is 1 CT scanner per 227,000 people, and in low-income countries, there is 1 per 1,694,000 people. This ratio differs drastically from HICs, where there is 1 per 25,000 people. ${ }^{15} \mathrm{CT}$ scans are essential for quickly identifying the need for acute neurosurgical intervention and providing visual guidance for operations. Although placement of EVDs is often performed using a blind freehand technique, rates of misplacement are high at $20 \%$ to $40 \%$, and incorporation of neurosurgical navigation techniques, such as CT or ultrasound guidance, can significantly increase the accuracy of EVD placement accuracy. ${ }^{16,17}$ Additionally, the development of robotic surgical devices has been shown to increase the accuracy of certain procedures, such as ventricular shunt placements. Lollis and Roberts demonstrated successful robotic placement of ventriculostomy catheters using preoperative imaging to plan the optimal catheter trajectory for placement. ${ }^{18}$ Robotic placement offers a unique opportunity for making EVD placement more widely available, especially in regions with limited access to a neurosurgeon. However, to allow surgeons without neurosurgical training to place EVDs, additional techniques and technology would have to be incorporated, which may rely on access to CT imaging.
In this article, we aim to highlight disparities in neurosurgical care, evaluate access to CT imaging around the world, and introduce a novel, inexpensive robotic navigation tool that has the potential to provide acute, lifesaving procedures in these underserved areas.

\section{Methods \\ Heat Map}

To visualize the regions in the world where a device would most benefit global neurosurgery assistive care, we compared the reported geographic concentration of neurosurgeons, CT scanners, incidence rates of indications for EVDs, and gross domestic product (GDP) on a heat map using the rworldmap package in $\mathrm{R}$ (The R Project). ${ }^{19}$

To build a heat map that superimposes the geographic concentrations of neurosurgeons, CT scanners, incidence rates of TBI-related death, meningitis, and pediatric congenital hydrocephalus, we extracted statistics from previous studies. ${ }^{20-24}$ Data regarding the number of neurosurgeons per country were obtained from the 2016 World Neurosurgery Workforce data published by the World Federation of Neurosurgical Societies. ${ }^{24}$ The GDP of countries was also taken into consideration, as this neurosurgical navigation technology aims to provide relief to countries that cannot afford to make improvements to their neurosurgical infrastructure..$^{25}$ The data from these sources were aggregated, and any country without a metric in the listed sources was excluded from further analysis.

The databases were aggregated, and the minimum and maximum statistics were scaled from 0 to 1 . Because this study aimed to implement an image-based neurosurgical navigation system for areas with low neurosurgical resources, despite the high incidence of EVD-indicating disease, areas dense in CT scanners and disease indications, and sparse in access to neurosurgery were compared.

\section{Indication Metric Equation}

One of the critical challenges in this study was developing a unified metric for the indication of the robotic navigation strategy incorporating CT availability, access to neurosurgeons, and medical indications for EVD placement. First, a weighted score was constructed for aggregating EVD indications based on the frequency of etiologies reported for EVD placement, labeled Place score $^{26}$ Place $_{\text {score }}$ $=\left(\mathrm{w}_{1} \times\right.$ Meningitis $)+\left(\mathrm{w}_{2} \times\right.$ Congenital Hydrocephalus $)+$ $\left(\mathrm{w}_{3} \times \mathrm{TBI}\right)$. Subsequently, Place ${ }_{\text {score }}$ was combined with the weighted importance of each of the other 3 features in the model, including the GDP of the country, inverse availability of neurosurgeons $\left(\mathrm{N}^{\prime}\right)$, and availability of CT scanners providing the indication for our system, labeled $\operatorname{Ind}_{\text {metric }}$ : $\operatorname{Ind}_{\text {metric }}=\left(\mathrm{w}_{4} \times \mathrm{GDP}\right)+\left(\mathrm{w}_{5} \times \mathrm{N}^{\prime}\right)+\left(\mathrm{w}_{6} \times\right.$ Place $\left._{\text {score }}\right)+\left(\mathrm{w}_{7}\right.$ $\times$ CT).

The weights $\left(\mathrm{w}_{4}-\mathrm{W}_{7}\right)$ were selected based on the relative incidence of the feature in describing the target population, encompassing $60 \%$ of the total weight for the unified metric (16\% CT availability, $42 \%$ lack of neurosurgery, and $42 \%$ low GDP). Countries with no CT scanners were weighted substantially lower than countries with few scanners. The indications for EVD accounted for the remaining $40 \%$ of the model weight ( $84 \%$ for TBI, $7 \%$ for 
meningitis, and $8 \%$ for hydrocephalus). These values were selected based on the relative usage of EVDs in the United States, providing a rough metric for how often EVDs would be used for certain conditions in other parts of the world. ${ }^{26}$

\section{Need Metric}

To consider where robotic navigation is most urgently needed, we altered the metric by setting the weight of CT and GDP to null. While CT scans are important for the implementation of our device, the need for navigation is dependent on the concentration of neurosurgeons and the incidence of indications for EVD placement. In future technologies, overcoming the need for CT scanners to truly overcome the full breadth of need may be explored. For this reason, we made an important distinction between the need for robotic navigation and the indications for our specific device.

\section{Robotic Implementation}

With the described limitations in neurosurgeon availability and the minimal necessary presence of CT imaging in LMICs, a cost-effective, passive navigation robot was designed to allow surgeons who are not trained in neurosurgery to place emergency EVDs in patients with acute or congenital hydrocephalus (Fig. 1A). The navigation system has 4 degrees of freedom from four 14-bit rotary encoders with an angular resolution of $0.022^{\circ}$ (CUI AMT22 Series). Mating adapters were designed with Fusion 360 (Autodesk) and 3D printed in polylactic acid with an Ender 5 3D printer (Creality). The adapters are connected to each encoder with supporting ball bearings and combined with machined aluminum rods to form the mechanical links between each robot joint. The robot base includes a stainless-steel bolt running through the bore of the base encoder that is used to securely attach the navigation system to the patient's skull.

The electrical components in the navigation system are based on a Raspberry Pi 0 WF microcontroller (Raspberry Pi Foundation) integrating the 4 rotary encoders connected by a custom-printed circuit board. The circuit board was designed in EasyEDA (EasyEDA), as shown in Fig. 2, and manufactured using the toner transfer method. ${ }^{27}$ Headers for the encoder pins were soldered to the circuit board, and a wiring harness was fabricated from 28 -gauge copper wire with standard terminals to connect the encoders, circuit board, and microcontroller generalpurpose input/output (GPIO) header.

During the operation, the microcontroller reads encoder positions and continuously sends the values over a wireless transmission control protocol/internet protocol (TCP/IP) connection to a separate laptop computer with the Ubuntu version 20.04 (Canonical Ltd.) operating system. The laptop coordinates robotic functions through the Robot Operating System (ROS) Noetic (Open Robotics), which wraps custom Python 3.8 (Python Software Foundation) scripts, enabling real-time surgical navigation for EVD placement. These functions include managing patient imaging, instructions for clinical setup, and a realtime navigation display for use during the procedure (Fig.

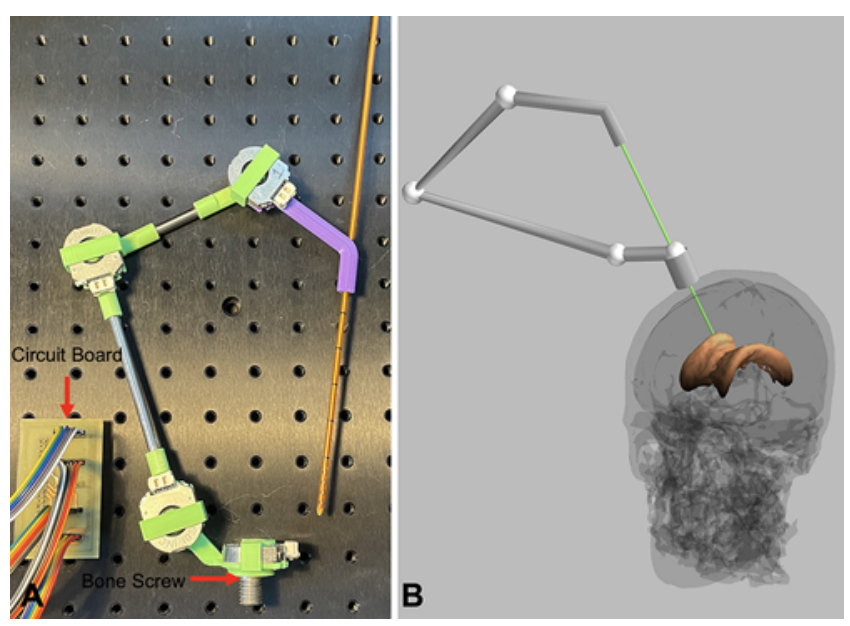

FIG. 1. Navigation concept. A: Prototype of the robotic navigation system with wiring harness removed for visibility. B: ROS-generated user interface for the robotic navigation system when attached and registered to a segmented head-neck CT.

1B). All software used is obtained from open-source packages to facilitate use in low-resource settings.

With trajectory-based navigation systems, accuracy and repeatability are critical to safe and effective operation. To verify the initial robot performance, an accuracy and repeatability assessment of the end-effector position was performed (the end effector is the interactive device at the end of the robotic arm). The robot base was rigidly fixed to a precision optical breadboard (MB4545/M, Thorlabs) with five 3D-printed calibration standoffs designed to determine the zero position of all encoders (Fig. 3A). Once calibrated, six 3D-printed targets were placed at varying distances from the base of the robot (Fig. 3B). These targets were designed to rigidly mate with the robot end effector and, when placed at known positions on the breadboard, served as ground truth positions for measuring robot accuracy. For each of these targets, the robot was moved from an arbitrary pose to the target, and the robot Cartesian coordinates were recorded. This was repeated a total of 5 times at each of the 6 targets, yielding 30 separate measurements. The difference in the Cartesian position was calculated from the observed robot measurements and expected ground truth positions. The resulting accuracy in each Cartesian direction $(\mathrm{X}, \mathrm{Y}, \mathrm{Z})$ was calculated as the average error in the given direction with repeatability calculated as the $95 \%$ CI (i.e., mean \pm 2 $\times \mathrm{SD})$. The overall system accuracy was calculated as the average Euclidean distance error between the measured and expected robot positions, and the repeatability was calculated as the $95 \% \mathrm{CI}$.

\section{Results \\ Heat Map Results}

Our query of the aforementioned databases yielded unified results usable for 169 countries. The top 25 countries indicated for general improvement in neurosurgical access to care (Table 1 and Fig. 4) is a ranking that considers the national numbers of neurosurgeons and the incidence of 


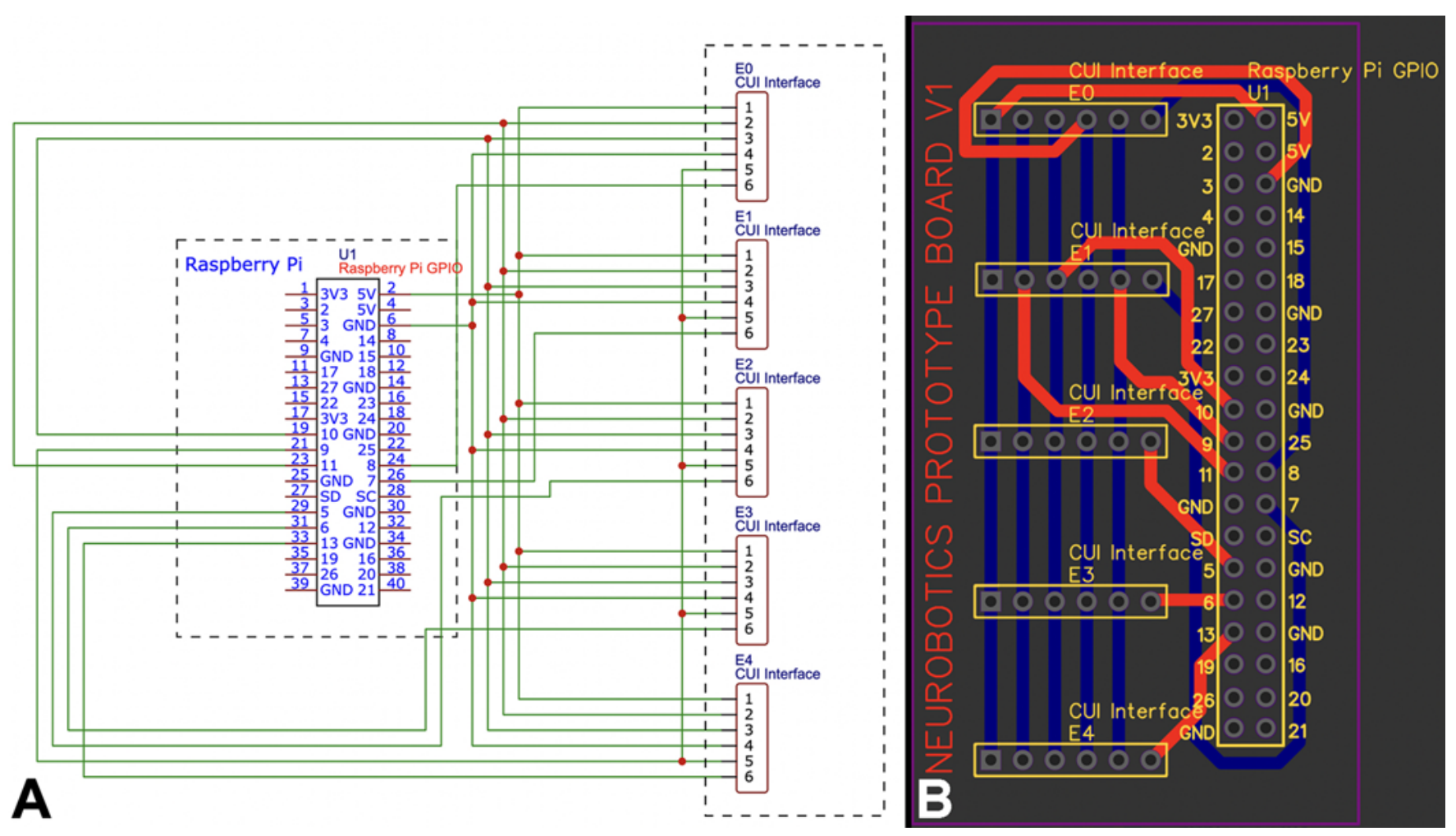

FIG. 2. Custom-printed circuit board wiring diagram. A: Pin-out wiring diagram with expansion port for a fifth encoder. B: The printed circuit board layout, which was printed using the toner transfer method.

the aforementioned indications for EVD placement. The top 25 countries indicated for the specific robotic navigation (Table 1 and Fig. 5) is a ranking that considers the national numbers of neurosurgeons, CT scanners, GDP,
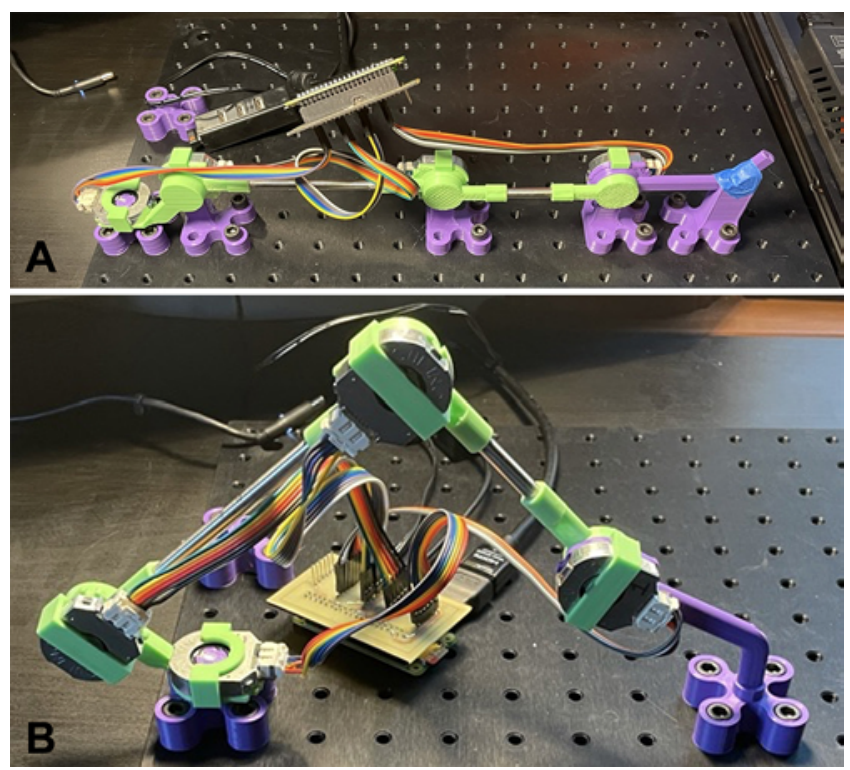

FIG. 3. Optical breadboard test setup. A: Calibration tool that uses the optical breadboard for setting "zero" positions of the 4 encoders. B: Optical breadboard targets for robotic repeatability and accuracy testing. and incidence of the aforementioned indications for EVD placement. We believe that this indication metric highlights environments with the capital and infrastructure to support a demonstrated need for the development of robotic navigation; the top 3 countries are Montenegro, Antigua and Barbuda, and Seychelles. Table 1 additionally demonstrates a ranking of countries based on the need metric. This need metric summarizes the general need for neurosurgical development regarding the placement of EVDs.

\section{Robotic Navigation Results}

The Cartesian position accuracy (X, Y, and Z translation) and overall accuracy of the robot were measured using the optical breadboard accuracy measurement system. The overall navigation system position accuracy was $1.53 \pm 2.50 \mathrm{~mm}(95 \% \mathrm{CI})$. The position accuracy in X was $-0.21 \pm 2.04 \mathrm{~mm}(95 \% \mathrm{CI})$. The position accuracy in $\mathrm{Y}$ was $0.12 \pm 1.43(95 \% \mathrm{CI})$. The position accuracy in $\mathrm{Z}$ was $-0.71 \pm 2.78 \mathrm{~mm}(95 \% \mathrm{CI})$. These results are visualized in Fig. 6.

\section{Discussion}

The model used to generate the Fig. 4 heat map was designed as a minimum metric necessary to observe the need for improved access to neurosurgical care globally, and to serve as a control for the model used to generate the Fig. 5 heat map, which was designed to compare indications and prevalence of care as they relate to the robotic navigation system proposed here. This device would be 
TABLE 1. Indications for improved access to neurosurgical care and navigated EVD placement, by country

\begin{tabular}{|c|c|c|}
\hline Rank & $\begin{array}{l}\text { Top } 25 \text { Countries Indicated for } \\
\text { Neurosurgical Development }\end{array}$ & $\begin{array}{l}\text { Top } 25 \text { Countries Indicated for } \\
\text { Navigated EVD Placement }\end{array}$ \\
\hline 1 & Montenegro & Montenegro \\
\hline 2 & Equatorial Guinea & Antigua \& Barbuda \\
\hline 3 & The Bahamas & Seychelles \\
\hline 4 & Suriname & The Bahamas \\
\hline 5 & Trinidad \& Tobago & Saint Vincent \& the Grenadines \\
\hline 6 & Saint Vincent \& the Grenadines & Trinidad \& Tobago \\
\hline 7 & Saint Lucia & Saint Lucia \\
\hline 8 & Antigua and Barbuda & Ireland \\
\hline 9 & Lesotho & Suriname \\
\hline 10 & Barbados & Bahrain \\
\hline 11 & Comoros & Malta \\
\hline 12 & Bahrain & Barbados \\
\hline 13 & Seychelles & Mauritius \\
\hline 14 & Sierra Leone & American Samoa \\
\hline 15 & Mauritius & Samoa \\
\hline 16 & American Samoa & Slovenia \\
\hline 17 & Liberia & Czechia \\
\hline 18 & Malta & Slovakia \\
\hline 19 & Ireland & Bulgaria \\
\hline 20 & Solomon Islands & Equatorial Guinea \\
\hline 21 & Samoa & Poland \\
\hline 22 & Syrian Arab Republic & Lesotho \\
\hline 23 & Slovenia & Comoros \\
\hline 24 & Czechia & Bosnia \& Herzegovina \\
\hline 25 & Slovakia & Sierra Leone \\
\hline
\end{tabular}

best utilized in a region with $\mathrm{CT}$ availability, a lack of neurosurgical care, and prevalent indications for placing an EVD. Countries with a lower GDP were also selected for, as we anticipated they would have a slower increase in access to care and, therefore, would benefit from a technology like this navigation system for a longer period of time.

While none of the countries are a perfect match for our device in all described metrics, some come close. Montenegro, for example, was shown to have a fairly developed medical system with many CT scanners; however, the country was in the lowest bracket for the number of neurosurgeons, despite moderate indications for EVDs. Montenegro, therefore, would be a country that could benefit from the implementation of this navigation system for lifesaving EVD indications, along with several other countries highlighted by our heat map.

This analysis demonstrates the paucity of CT scanners in areas where the indications for EVDs are high. Additionally, even in low-income countries, the presence of CT scanners is more likely to be in higher-income areas. ${ }^{14,28}$ Given the current necessity of CT in ascertaining the reference image for navigating the robot, this analysis demonstrates a critical limitation and restriction of implementation, which can be extrapolated to any navigation assistance technology for EVD placement.

With sufficient availability of the necessary CT scanners, lack of neurosurgical care, and a high incidence of acute TBI, there is an evidenced need for assistive technology to enable lifesaving treatment in these regions. To meet this demand, the presented navigation system was designed with a clinical workflow, which is an adaptation of the current standard of care (Fig. 7). ${ }^{29-31}$ This procedure begins with a preoperative CT scan that is subsequently segmented using the open-source 3D Slicer program (3D Slicer, slicer.org) to identify the skull for robot CT

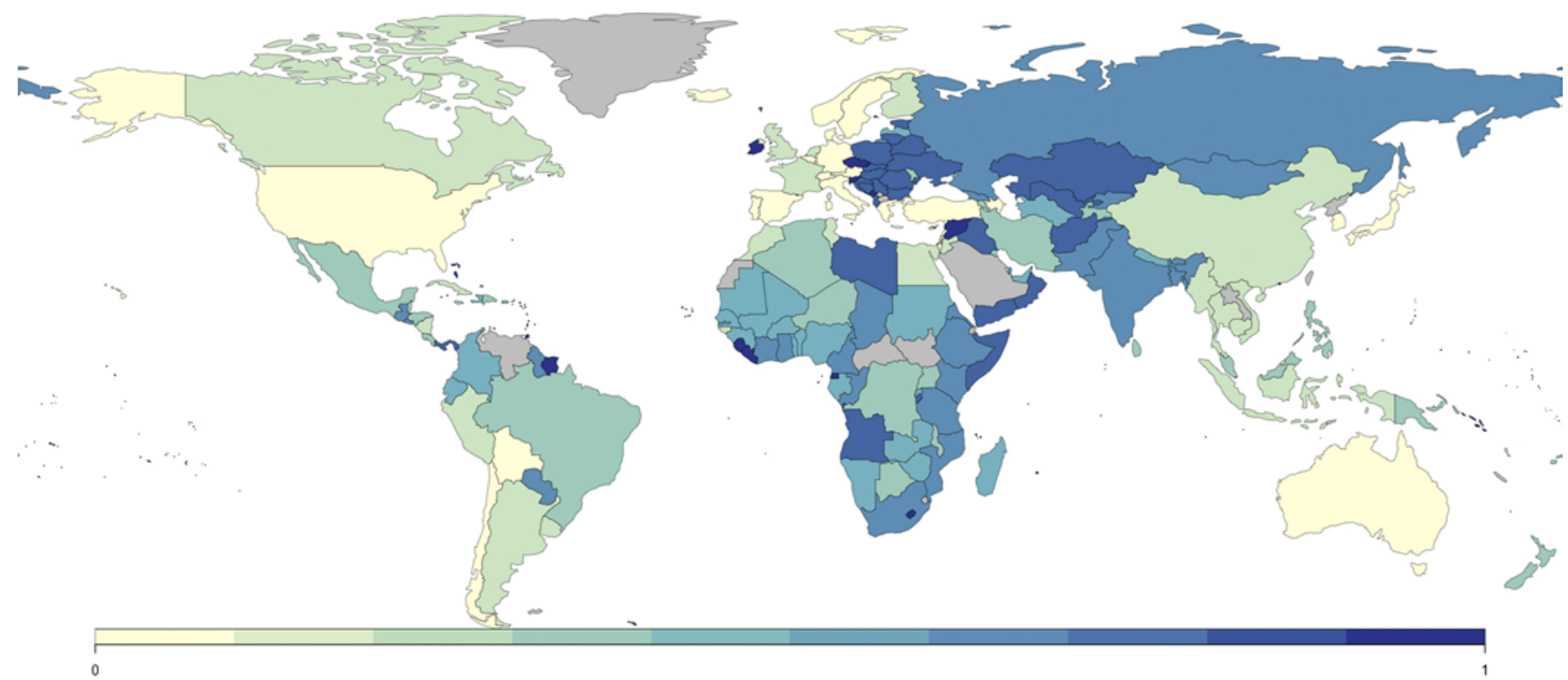

FIG. 4. Heat map showing countries with a need for neurosurgeons and indications for EVD. By combining worldwide distributions of neurosurgeons and the incidence of TBI, meningitis, and hydrocephalus, this map indicates where the need for a robotic navigation system is highest, with 0 indicating low need and 1 indicating high need. Made in $\mathrm{R}$ using the rworldmap package. ${ }^{19}$ 


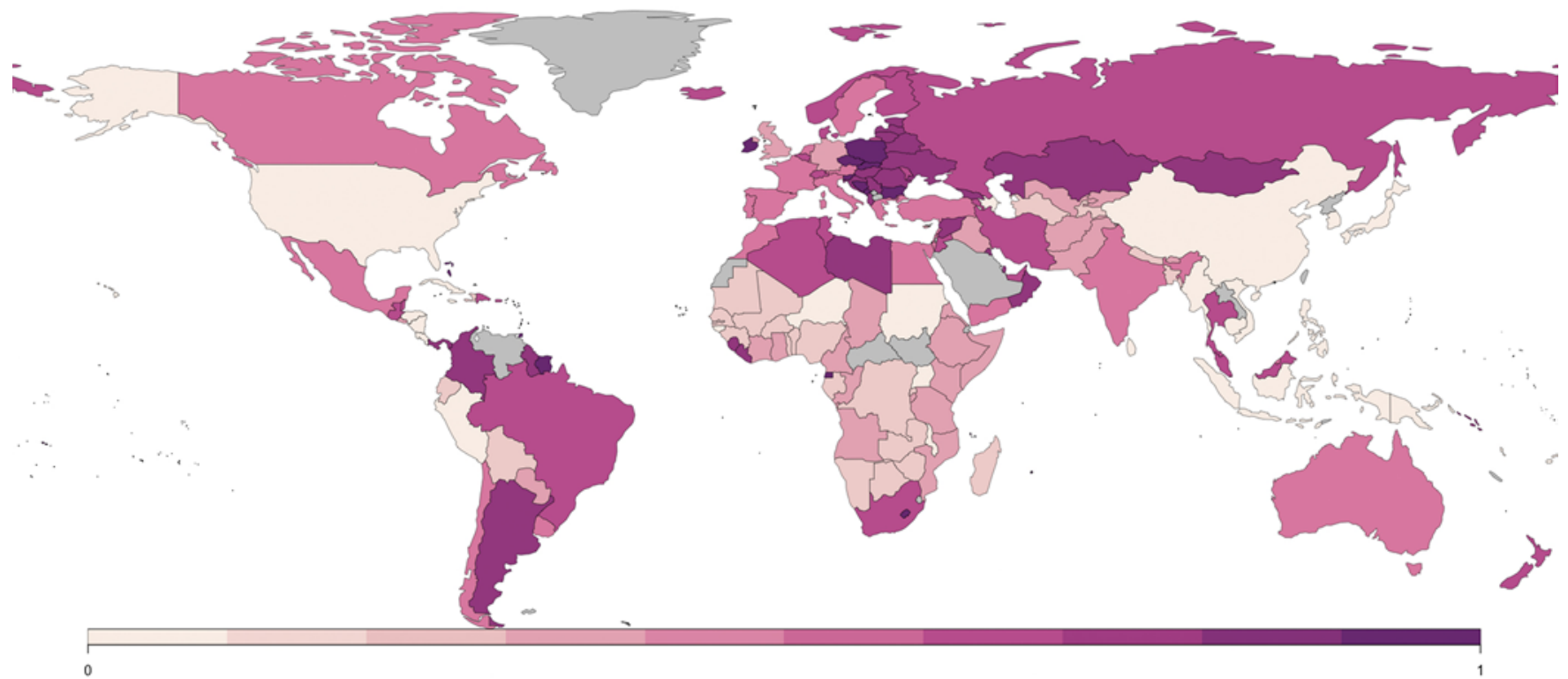

FIG. 5. Heat map showing indications for device trials by weighting the density of neurosurgeons, CT scanners, GDP, and the incidence of TBI-related death, meningitis, and pediatric congenital hydrocephalus. Scores range from 0 to 1 , relaying minor to major benefit of device trials by country. Made in R using the rworldmap package..$^{19}$

registration and the ventricles for generating target trajectory. The surgeon then places a burr hole at Kocher's point $(10-12 \mathrm{~cm}$ posterior to the eye and $2-3 \mathrm{~cm}$ lateral from the midline) where the robot attaches, using the integrated bone screw (Fig. 1A). After securely attaching the robot to the patient, a rigid registration is performed in the custom software to relate the robot and preoperative CT coordinate spaces. Once registration is complete, the user interface displays the real-time position of the robot and attached EVD, providing a continuous view of the trajectory of the EVD relative to the segmented ventricles for surgical guidance. Following the workflow, the patient will be monitored and ideally transferred to a care team that can better manage their primary neurological condition.

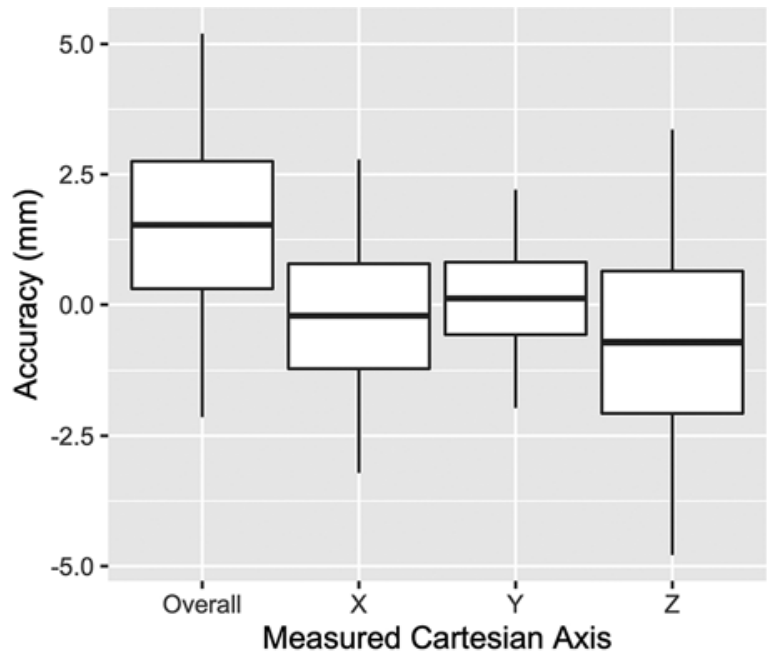

FIG. 6. Boxplots showing overall robotic accuracy for 30 samples with Cartesian (X, Y, and Z) coordinate accuracy for comparison.
The initial accuracy and repeatability measurements collected from the robot suggest that a low-cost approach to robotic neurosurgery is feasible. A previous study found that the average atrial diameter of fetal lateral ventricles is $7.6 \mathrm{~mm}$ and the ventricle size will only increase with age. ${ }^{32}$ With an overall navigation system accuracy of 1.53 $\pm 2.50 \mathrm{~mm}(95 \% \mathrm{CI})$, it will be capable of successfully guiding EVD placement, even in challenging pediatric lateral ventricles as in the case of congenital hydrocephalus. One limitation in the application of the robot is the current need for bone screws in the skull, which would render this more difficult for use in infants. Berry et al. reported depressed cranial fractures, epidural and subdural hematomas, and CSF leakage as potential complications with use in children younger than 10 years. ${ }^{33,34}$ To avoid undue risk, the current robotic implementation would be used in patients 10 years or older, and investigation into the use of an alternative fixation method will be required to use this technology in younger patients.

With an accurate low-cost robot that is easily modified due to its simple mechanical design and open-source software, applications can be readily expanded to include other navigation-based procedures. For example, navigation is useful for accessing lesions or cysts that reside deeper in the brain, as superficial landmarks may not be as accurate in these cases. ${ }^{35}$ It may also be helpful in deep brain stimulation surgeries for certain neurological disorders, such as Parkinson's disease, dystonia, chronic pain, and seizure disorder diagnosis with stereoelectroencephalography, where imaging is required to determine the target locations for electrode lead placement. ${ }^{36,37}$ Accurate, low-cost robots also may be useful in placing other types of CSF shunts, such as ventriculoperitoneal and ventriculosubgaleal shunts, and ventricular access devices. ${ }^{38}$ Expanding beyond neurosurgery, the passive navigation robot could be used for procedures such as percutaneous biopsies of 
Standard of Care EVD Placement Workflow

\begin{tabular}{|c|}
\hline Preoperative imaging to assess landmarks \\
\hline - Identify the ipsilateral medial canthus and ipsilateral tragus \\
\hline Prepare patient \\
\hline $\begin{array}{l}\text { - Place patient in supine position with bed positioned at } 45 \\
\text { degrees } \\
\text { - Place absorbent pad underneath patient's head } \\
\text { - Wash scalp with soap and water and dry with towel } \\
\text { - Clip hair on frontal scalp with clippers } \\
\text { - Use pen and ruler to mark a linear, } 3 \mathrm{~cm} \text { incision at the } \\
\text { EVD entry point on skull, } 10-12 \mathrm{~cm} \text { posterior to eye and 2- } \\
3 \mathrm{~cm} \text { lateral to midline of skull } \\
\text { - Secure patient's head to bed with } 3 \text {-in silk tape }\end{array}$ \\
\hline Prepare sterile site \\
\hline $\begin{array}{l}\text { Drape surgical table with sterile sheet } \\
\text { - Upen cranial access and EVD kits } \\
\text { marked incision site } \\
\text { - Drape with full body fenestrated drape } \\
\text { - Inject local anesthetic along marked surgical site }\end{array}$ \\
\hline Preparing for EVD Insertion \\
\hline $\begin{array}{l}\text { Make an incision at procedural site down to the bone } \\
\text { - Create burr hole with hand drill, removing loose pieces of } \\
\text { bone with forceps } \\
\text { - Pierce dura multiple times with spinal needle }\end{array}$ \\
\hline Insert and secure EVD \\
\hline $\begin{array}{l}\text { - Advance trocar inferiorly to incision, emerging } 3-5 \mathrm{~cm} \\
\text { lateral and posterior to incision } \\
\text { - Insert stylet into catheter } \\
\text { - Advance catheter in trajectory into frontal horn of lateral } \\
\text { ventricle perpendicular to skull towards landmarks } \\
\text { - Remove stylet \& connect distal end of catheter to serrated } \\
\text { end of trocar } \\
\text { - Secure catheter to skin with suture } \\
\text { - Cut distal end of catheter close to trocar } \\
\text { Remove retractor and close incision with running or } \\
\text { continuous suture }\end{array}$ \\
\hline
\end{tabular}

\section{Connect EVD to Drain and ICP monitor}

- Connect catheter to sterile tubing from collecting system

- Adjust height of collecting system and set transducer to zero; monitor ICP

- Set height of collecting system to desired level depending on condition treated

- Dress wound with antibiotic impregnated disc (chlorhexidine patch) and sterile transparent film dressing

\section{Proposed Navigation Device Assisted EVD Placement Workflow}

\section{Preoperative imaging to assess landmarks}

- Upload scan into EVD Software

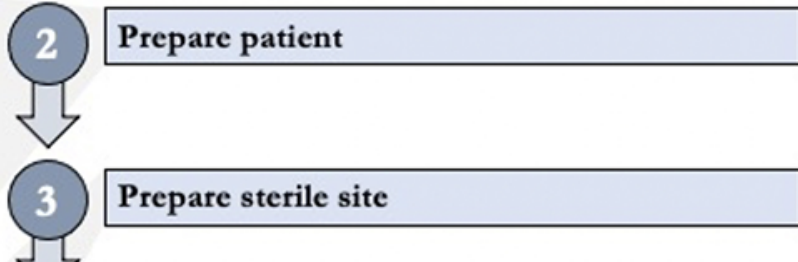

4 Preparing for EVD Insertion under Navigation

- Make an incision at procedural site down to the bone

- Create burr hole with hand drill, removing loose pieces of bone with forceps

- Pierce dura multiple times with spinal needle

- Mount navigation device in burr hole

\begin{tabular}{ll|} 
Patient Registration \\
\hline $\begin{array}{l}\text { Use probe to create surface mapping of points on patient } \\
\cdot\end{array}$ & Points registered and mapped on pre-operative imaging \\
$\cdot$ & Verify registration
\end{tabular}

\section{6) Trajectory Planning and EVD Insertion}

- Advance trocar inferiorly to incision, emerging $3-5 \mathrm{~cm}$ lateral and posterior to incision

- Insert stylet into catheter

- Attach stylet to navigation device

- Guide the navigation device by hand toward the incision site

- Viewing the software, guide the catheter toward the highlighted ventricles on the patient image

- the ventricles will highlight in green when aligned with the trajectory of the catheter, guiding the surgeon to the correct location, and red when misaligned

- Remove stylet (still attached to the navigation device)

- Cut distal end of catheter close to trocar

- Remove retractor and close incision with running or continuous suture

\section{Connect EVD to Drain and ICP monitor}

FIG. 7. Workflow comparison between standard-of-care EVD placement and the proposed navigation device-assisted EVD placement. 
deep structures (e.g., lungs, kidney, or liver), placing pedicle screws for spine surgery, or even as a surgical training device to provide continuous trajectory feedback for novice surgeons.

\section{Limitations of the Heat Map}

In total, 169 countries were included in the heat map. The countries that were not represented in the data are represented in gray on the heat map. Additionally, although the relative weights for each component of $\operatorname{Ind}_{\text {metric }}$ were selected a priori, the rationale for the values for lack of access to neurosurgery, availability of CT, GDP, and the combined indications for EVD placement were dependent on subjective factors based on discussion of the device with the design and implementation team. This creates the possibility for bias in the feasibility analysis.

\section{Limitations of the Robotic Navigation System}

The navigation system was limited by several factors: the accuracy of the encoders, the rigidity of the 3D-printed parts, and the cost of the components. With the specifications of the encoders, it is theoretically estimated that the worst-case error is $<2.44 \mathrm{~mm}$. If the parts were machined or cast instead of 3D printed, they could be made far more rigid, leading to further reductions in the observed errors associated with flexion and tolerance issues inherent to $3 \mathrm{D}$ printing. Finally, the costs associated with this robot were intentionally minimized in order to make this technology more feasible to implement in low-resource environments. The prototype cost was $\$ 249.46$, including all electronics and raw materials and excluding any tools used in production as well as the Codman Bactiseal EVD (Integra LifeSciences) and optical breadboard pictured in Fig. 2. This cost could potentially be reduced further by manufacturing the system to scale.

\section{Future Directions}

While this study informs on the feasibility of robotics in EVD navigation globally, there are important obstacles to implementation of this navigation system. Despite verifying robot accuracy and demonstrating that it meets the standard of care for technology used in HICs, the system must be deployed in a representative clinical model to validate the clinical use. First, a cadaver study will assist with quantifying the efficacy and workflow of this prototype robot for navigation in humans. This cadaver study will use Thiel's embalming method with intracerebral, ventricular formalin-injected specimens, which has been shown to be effective for EVD simulation. ${ }^{39}$ Following cadaveric testing, prototype design improvements, including minimization of external wiring, creation of a disposable sterile sleeve, a battery-powered wireless implementation, and others, will be made to finalize the minimum viable product.

Additional modalities may also be investigated for navigation and imaging, including electrode placement, brain biopsy, and other trajectory-based burr hole procedures. Military or emergency medical service trauma environments will additionally be explored. The use of CT is required; however, ongoing investigations of low- cost, noninvasive lateral ventricular imaging may serve as a valuable addition to our proposed navigation system to provide reference imaging for EVD placement. ${ }^{40,41}$

\section{Conclusions}

The lack of access to EVDs may preclude a patient to any type of care for the management of hydrocephalus. While lifesaving measures are performed in some circumstances by midlevel practitioners with similar success, a navigation system could substantially improve the confidence of the practitioner in high-stress situations. ${ }^{42} \mathrm{We}$ sought to create a device that bridged an important skillsassociated obstacle for emergency neurosurgery for those surgeons who are without explicit neurosurgical training. Using this device's user interface, a surgeon will have the tools and guidance to safely perform EVD placement. In this feasibility study, we find that implementing such a device is feasible and may be impactful to specific countries.

\section{Acknowledgments}

This research was supported by an Innovation Pathway Grant from the Carle Illinois College of Medicine.

We thank the Department of Neurosurgery at Saint Louis University for initial contributions to this work; in particular, Richard Bucholz, MD; Philippe Mercier, MD, PhD; and Najib El Tecle, MD. In addition, we thank Dr. Andrew Hall, ScD; and Michael Borovik, MS, for their support in the development of this concept.

\section{References}

1. Muralidharan R. External ventricular drains: management and complications. Surg Neurol Int. 2015;6(6)(suppl 6):S271S274.

2. Greenberg MS. Handbook of Neurosurgery. 9th ed. Thieme; 2020.

3. Chau CYC, Craven CL, Rubiano AM, et al. The evolution of the role of external ventricular drainage in traumatic brain injury. J Clin Med. 2019;8(9):1422.

4. Dewan MC, Rattani A, Gupta S, et al. Estimating the global incidence of traumatic brain injury. J Neurosurg. 2018;130(4): 1080-1097.

5. Dewan MC, Rattani A, Fieggen G, et al. Global neurosurgery: the current capacity and deficit in the provision of essential neurosurgical care. J Neurosurg. 2018;130(4):10551064.

6. Qalab A, Asad R, Hakeem M, Ahmad M, Haq A. Paediatric external ventricular drains: experience from a tertiary care hospital of a developing country. J Pak Med Assoc. 2016; 66(Suppl 3)(10):S72-S74.

7. Ojo OA, Asha MA, Bankole OB, Kanu OO. Improvised external ventricular drain in neurosurgery: a Nigerian tertiary hospital experience. J Neurosci Rural Pract. 2015;6(3):304308.

8. Glimåker M, Johansson B, Halldorsdottir H, et al. Neurointensive treatment targeting intracranial hypertension improves outcome in severe bacterial meningitis: an intervention-control study. PLoS One. 2014;9(3):e91976.

9. Tariq A, Aguilar-Salinas P, Hanel RA, Naval N, Chmayssani M. The role of ICP monitoring in meningitis. Neurosurg Focus. 2017;43(5):E7.

10. Tully HM, Dobyns WB. Infantile hydrocephalus: a review of epidemiology, classification and causes. Eur J Med Genet. 2014;57(8):359-368.

11. Bassan H, Eshel R, Golan I, et al. Timing of external ventric- 
ular drainage and neurodevelopmental outcome in preterm infants with posthemorrhagic hydrocephalus. Eur J Paediatr Neurol. 2012;16(6):662-670.

12. Miller T. Ensuring an adequate neurosurgical workforce for the 21st century. AANS Neurosurgeon. November 2, 2016. Accessed November 17, 2021. https://aansneurosurgeon. org/departments/neurosurgical-workforce-shortage-effectsubspecialization-cast-shortening-residency-training/

13. Punchak M, Mukhopadhyay S, Sachdev S, et al. Neurosurgical care: availability and access in low-income and middleincome countries. World Neurosurg. 2018;112:e240-e254.

14. Mansouri A, Ku JC, Khu KJ, et al. Exploratory analysis into reasonable timeframes for the provision of neurosurgical care in low- and middle-income countries. World Neurosurg. 2018;117:e679-e691.

15. Hricak H, Abdel-Wahab M, Atun R, et al. Medical imaging and nuclear medicine: a Lancet Oncology Commission. Lancet Oncol. 2021;22(4):e136-e172.

16. AlAzri A, Mok K, Chankowsky J, Mullah M, Marcoux J. Placement accuracy of external ventricular drain when comparing freehand insertion to neuronavigation guidance in severe traumatic brain injury. Acta Neurochir (Wien). 2017; 159(8):1399-1411.

17. Wilson TJ, Stetler WR Jr, Al-Holou WN, Sullivan SE. Comparison of the accuracy of ventricular catheter placement using freehand placement, ultrasonic guidance, and stereotactic neuronavigation. J Neurosurg. 2013;119(1):66-70.

18. Lollis SS, Roberts DW. Robotic catheter ventriculostomy: feasibility, efficacy, and implications. J Neurosurg. 2008; 108(2):269-274.

19. South A. rworldmap: a new $\mathrm{R}$ package for mapping global data. $R$ J. 2011;3(1):35-45.

20. James SL, Theadom A, Ellenbogen RG, et al. Global, regional, and national burden of traumatic brain injury and spinal cord injury, 1990-2016: a systematic analysis for the Global Burden of Disease Study 2016. Lancet Neurol. 2019; 18(1):56-87.

21. Zunt JR, Kassebaum NJ, Blake N, et al. Global, regional, and national burden of meningitis, 1990-2016: a systematic analysis for the Global Burden of Disease Study 2016. Lancet Neurol. 2018;17(12):1061-1082.

22. Mukhopadhyay S, Punchak M, Rattani A, et al. The global neurosurgical workforce: a mixed-methods assessment of density and growth. J Neurosurg. 2019;130(4):1142-1148.

23. IMAGINE - CT scanners (per 1 mil). IAEA Human Health Campus. Accessed November 17, 2021. https://humanhealth. iaea.org/HHW/DBStatistics/IMAGINEMaps2.html

24. Global Neurosurgical Workforce Map - 2016 World Neurosurgery Workforce. World Federation of Neurosurgical Societies. Accessed November 17, 2021. https://wfns.org/menu/61/ global-neurosurgical-workforce-map

25. GDP by country. Worldometer. Accessed August 31, 2021. https://www.worldometers.info/gdp/gdp-by-country/

26. Rosenbaum BP, Vadera S, Kelly ML, Kshettry VR, Weil RJ. Ventriculostomy: frequency, length of stay and in-hospital mortality in the United States of America, 1988-2010. J Clin Neurosci. 2014;21(4):623-632.

27. Easley CJ, Benninger RKP, Shaver JH, Head WS, Piston DW. Rapid and inexpensive fabrication of polymeric microfluidic devices via toner transfer masking. Lab Chip. 2009;9(8):11191127.

28. Silverstein J. Most of the world doesn't have access to Xrays. The Atlantic. Published September 27, 2016. Accessed November 17, 2021. https://www.theatlantic.com/health/ archive/2016/09/radiology-gap/501803/

29. External ventricular drain placement stealth study. NCT03696043. ClinicalTrials.gov. Last updated June 8, 2021. Accessed November 17, 2021. https://clinicaltrials.gov/ct2/ show/NCT03696043
30. Dossani RH, Patra DP, Terrell DL, Willis B. Placement of an external ventricular drain. $N$ Engl J Med. 2021;384(2):e3.

31. Eftekhar B. App-assisted external ventricular drain insertion. J Neurosurg. 2016;125(3):754-758.

32. Cardoza JD, Goldstein RB, Filly RA. Exclusion of fetal ventriculomegaly with a single measurement: the width of the lateral ventricular atrium. Radiology. 1988;169(3):711-714.

33. Wong WB, Haynes RJ. Osteology of the pediatric skull. Considerations of halo pin placement. Spine (Phila Pa 1976). 1994;19(13):1451-1454.

34. Berry C, Sandberg DI, Hoh DJ, Krieger MD, McComb JG. Use of cranial fixation pins in pediatric neurosurgery. Neurosurgery. 2008;62(4):913-919.

35. Schulz C, Waldeck S, Mauer UM. Intraoperative image guidance in neurosurgery: development, current indications, and future trends. Radiol Res Pract. 2012;2012:197364.

36. Perlmutter JS, Mink JW. Deep brain stimulation. Annu Rev Neurosci. 2006;29:229-257.

37. Dormont D, Seidenwurm D, Galanaud D, Cornu P, Yelnik J, Bardinet E. Neuroimaging and deep brain stimulation. AJNR Am J Neuroradiol. 2010;31(1):15-23.

38. Kuo MF. Surgical management of intraventricular hemorrhage and posthemorrhagic hydrocephalus in premature infants. Biomed J. 2020;43(3):268-276.

39. Miyake S, Suenaga J, Miyazaki R, et al. Thiel's embalming method with additional intra-cerebral ventricular formalin injection (TEIF) for cadaver training of head and brain surgery. Anat Sci Int. 2020;95(4):564-570.

40. Shibata Y, Kruskal JB, Palmer MR. Imaging of cerebrospinal fluid space and movement of hydrocephalus mice using near infrared fluorescence. Neurol Sci. 2007;28(2):87-92.

41. Lange F, Tachtsidis I. Clinical brain monitoring with time domain NIRS: a review and future perspectives. Appl Sci (Basel). 2019;9(8):1612.

42. Ellens NR, Fischer DL, Meldau JE, Schroeder BA, Patra SE. External ventricular drain placement accuracy and safety when done by midlevel practitioners. Neurosurgery. 2019; 84(1):235-241.

\section{Disclosures}

Conception and design: AD Smith, Teague. Acquisition of data: AD Smith, Teague, Naik, Janbahan, Krist. Analysis and interpretation of data: AD Smith, Teague, Naik, Janbahan, EJ Smith, Krist. Drafting the article: AD Smith, Naik, Janbahan, EJ Smith, Krist. Critically revising the article: Hassaneen, AD Smith, Naik, Janbahan, EJ Smith, Krist, Parupalli. Reviewed submitted version of manuscript: all authors. Approved the final version of the manuscript on behalf of all authors: Hassaneen. Statistical analysis: AD Smith, Teague, Naik, Janbahan. Administrative/technical/ material support: Hassaneen, AD Smith, Teague, EJ Smith. Study supervision: Hassaneen, AD Smith, Naik, Teal.

\section{Supplemental Information \\ Previous Presentations}

Portions of this paper were presented orally at the 16th Annual Coordinated Sciences Laboratory Student Conference, University of Illinois at Urbana-Champaign, April 9, 2021.

\section{Correspondence}

Wael Hassaneen: Neuroscience Institute, Carle Foundation Hospital, Urbana, IL.wael.mostafa@carle.com. 